\title{
Quality assessment of solar UV irradiance measured with array spectroradiometers
}

\author{
Luca Egli $^{1}$, Julian Gröbner ${ }^{1}$, Gregor Hülsen ${ }^{1}$, Luciano Bachmann $^{2}$, Mario Blumthaler ${ }^{3}$, Jimmy Dubard $^{4}$, \\ Marina Khazova $^{5}$, Richard Kift ${ }^{6}$, Kees Hoogendijk ${ }^{7}$, Antonio Serrano ${ }^{8}$, Andrew Smedley ${ }^{6}$, and \\ José-Manuel Vilaplana ${ }^{9}$ \\ ${ }^{1}$ Physikalisch Meteorologisches Observatorium and World Radiation Center (PMOD/WRC), \\ Davos, Switzerland \\ ${ }^{2}$ Faculdade de Filosofia, Ciências e Letras de Ribeirão Preto Universidade de São Paulo, São Paulo, Brazil \\ ${ }^{3}$ Medical University Innsbruck, Biomedical Physics, Innsbruck, Austria \\ ${ }^{4}$ Laboratoire national de métrologie et d'essais LNE, Paris, France \\ ${ }^{5}$ Public Health England, Chilton, UK \\ ${ }^{6}$ School of Earth Atmospheric and Environmental Sciences, University of Manchester, Manchester, UK \\ ${ }^{7}$ EKO INSTRUMENTS Europe B.V., Delft, the Netherlands \\ ${ }^{8}$ Department of Physics, University of Extremadura, Badajoz, Spain \\ ${ }^{9}$ Instituto Nacional de Técnica Aeroespacial - INTA, El Arenosillo, Spain
}

Correspondence to: Luca Egli (luca.egli@pmodwrc.ch)

Received: 26 November 2015 - Published in Atmos. Meas. Tech. Discuss.: 21 December 2015

Revised: 11 March 2016 - Accepted: 15 March 2016 - Published: 8 April 2016

\begin{abstract}
The reliable quantification of ultraviolet (UV) radiation at the earth's surface requires accurate measurements of spectral global solar UV irradiance in order to determine the UV exposure to human skin and to understand long-term trends in this parameter. Array spectroradiometers (ASRMs) are small, light, robust and cost-effective instruments, and are increasingly used for spectral irradiance measurements. Within the European EMRP ENV03 project "Solar UV", new devices, guidelines and characterization methods have been developed to improve solar UV measurements with ASRMs, and support to the end user community has been provided. In order to assess the quality of 14 end user ASRMs, a solar UV intercomparison was held on the measurement platform of the World Radiation Center (PMOD/WRC) in Davos, Switzerland, from 10 to 17 July 2014. The results of the blind intercomparison revealed that ASRMs, currently used for solar UV measurements, show a large variation in the quality of their solar UV measurements. Most of the instruments overestimate the erythema-weighted UV index - in particular at large solar zenith angles - due to stray light contribution in the UV-B range. The spectral analysis of global solar UV irradiance further supported the finding that the un-
\end{abstract}

certainties in the UV-B range are very large due to stray light contribution in this wavelength range. In summary, the UV index may be detected by some commercially available ASRMs within 5\% compared to the world reference spectroradiometer, if well characterized and calibrated, but only for a limited range of solar zenith angles. Generally, the tested instruments are not yet suitable for solar UV measurements for the entire range between 290 and $400 \mathrm{~nm}$ under all atmospheric conditions.

\section{Introduction}

Solar UV radiation, in particular UV-B in the wavelength range between 290 and $315 \mathrm{~nm}$, is of great biological importance because photons in this region may damage deoxyribonucleic acid (DNA) molecules and some proteins of living organisms. An increase in surface UV radiation could cause additional non-melanoma and melanoma skin cancers (http://www.who.int/uv/uv_and_health/en/index.html). On the other hand, UV-B is essential for the synthesis of vitamin $\mathrm{D}_{3}$ in the human body, which has beneficial health 
effects and helps in the prevention of some diseases (Holick et al., 2006; Gröbner et al., 2015). The knowledge of the entire UV spectrum from 290 to $400 \mathrm{~nm}$ is essential to weigh the measured spectrum with different action spectra, such as erythema, vitamin $\mathrm{D}_{3}$ and DNA damage (e.g. McKinlay and Diffey, 1987; Anders et al., 1995), which in turn are needed to better inform the public about the different critical doses and their impact on human health. Measurements of UV spectra in the aforementioned spectral range can also be converted to actinic flux spectra (e.g. Kazadzis et al., 2000) to investigate photochemical processes in the atmosphere in terms of photolysis frequencies (e.g. Hofzumanhaus et al., 1999; Edwards and Monks, 2003).

In order to understand long-term trends of solar UV radiation at the earth's surface for human health protection and to test radiative atmospheric transfer models (e.g. libRadtran, Mayer and Kylling, 2005), accurate instruments for measuring global solar spectral UV irradiance are required. The current knowledge on spectral solar UV radiation is limited to very few places worldwide, where spectral solar UV monitoring instruments are located. Commonly, UV irradiance is observed with scanning spectroradiometers measuring the solar irradiance spectrum sequentially, which requires several minutes of scanning time and instruments that are cost- and maintenance-intensive (Bittar and McKenzie, 1990; Bernhard et al., 1997; Bais et al., 2001). The interpretation of UV data from scanning spectroradiometers is difficult in the case of fast-varying atmospheric conditions (e.g. moving cloud). Additionally, the slow scanning speed does not allow fast sampling rates, limiting the number of measurements available per day. Large-scale deployment of such instruments is limited by the required manpower and infrastructure to guarantee an adequate level of uncertainty. UV monitoring at additional locations, however, is desired to better understand the relationship between UV radiation and influencing factors on human health.

Array spectroradiometers (ASRMs) are widely used in different applications in science and operational monitoring (e.g. Coleman et al., 2008; Seckmeyer et al., 2010). They provide the advantages of fast data acquisition measuring entire spectra quasi-simultaneously on the order of seconds. Compared to scanning spectroradiometer systems, ASRMs are small, light, portable, without moving parts and mostly costefficient, and may therefore be the next generation of instruments to increase the worldwide network of spectral solar UV measurements. However, available ASRMs are limited for accurate solar UV measurements without complex correction methodologies (Nevas et al., 2014), due to the large dynamic range of the solar UV radiation between 290 and $400 \mathrm{~nm}$ caused by the sharp cut-off of the solar UV spectrum as a consequence of stratospheric ozone absorption. ASRMs are single monochromator instruments, suffering significantly from stray light contamination, thereby biasing irradiance measurements at short wavelengths in the UV-B. Furthermore, the silicon-based array detectors exhibit lower sensitivity than photomultipliers commonly used in scanning spectroradiometers, which makes it difficult to detect the low irradiance level at short wavelengths. This is a severe problem for measurements of solar UV radiation and it is therefore important to (a) follow strict procedures when using ASRMs for measuring spectral solar UV radiation, (b) characterize and correct for stray light, (c) calibrate the wavelength dependency and (d) determine the linearity of the entire system (Seckmeyer et al., 2010).

Within the European EMRP ENV03 project "Solar UV" (http://projects.pmodwrc.ch/env03/index.php), new devices, guidelines and characterization methods have been developed to improve solar UV measurements of ASRMs, and to provide support to the end user community (Blumthaler et al., 2013). One major dissemination activity at the end of the EMRP project was the UV intercomparison held on the measurement platform at the World Radiation Center (PMOD/WRC) in Davos, Switzerland from 10 to 17 July 2014, attended by project partners and external participants from the end user community. The aim of the intercomparison was to provide identical characterization and calibration services (e.g. standard lamp for spectral irradiance) for the participants and to assess the quality of ASRMs specifically designed for solar UV measurements in a blind intercomparison, comparing the data as provided by the end users with a reference instrument (as in Bais et al., 2001). This work demonstrates the limitation of ASRMs for detecting global solar UV radiation. Based on this study, the future aim of the World Calibration Center for UV radiation (WCCUV) at PMOD/WRC, operating on behalf of the World Meteorological Organization (WMO), is to further improve the quality of spectral solar UV measurements derived from ASRMs.

\section{Data and methods}

For accurate measurements of the global solar UV irradiance in the existing monitoring network, high quality instrumentation is currently in operation. Commonly, these instruments are scanning spectroradiometers, consisting of a double monochromator and a photomultiplier as detector. The double monochromator set-up ensures sufficient stray light reduction to cover 5-6 orders of magnitude of irradiance levels, while the photomultiplier is able to detect irradiance levels as low as $10^{-6} \mathrm{~W} \mathrm{~m}^{-2} \mathrm{~nm}^{-1}$.

At the intercomparison two double monochromator spectroradiometers were operated as reference instruments in order to assess the quality of ASRMs: (1) QASUME (quality assurance of spectral solar UV measurements in Europe; Gröbner et al., 2005), which was the reference for the intercomparison and (2) QASUME II, which should demonstrate measurements of solar UV with a double monochromator instrument compared to ASRMs. 


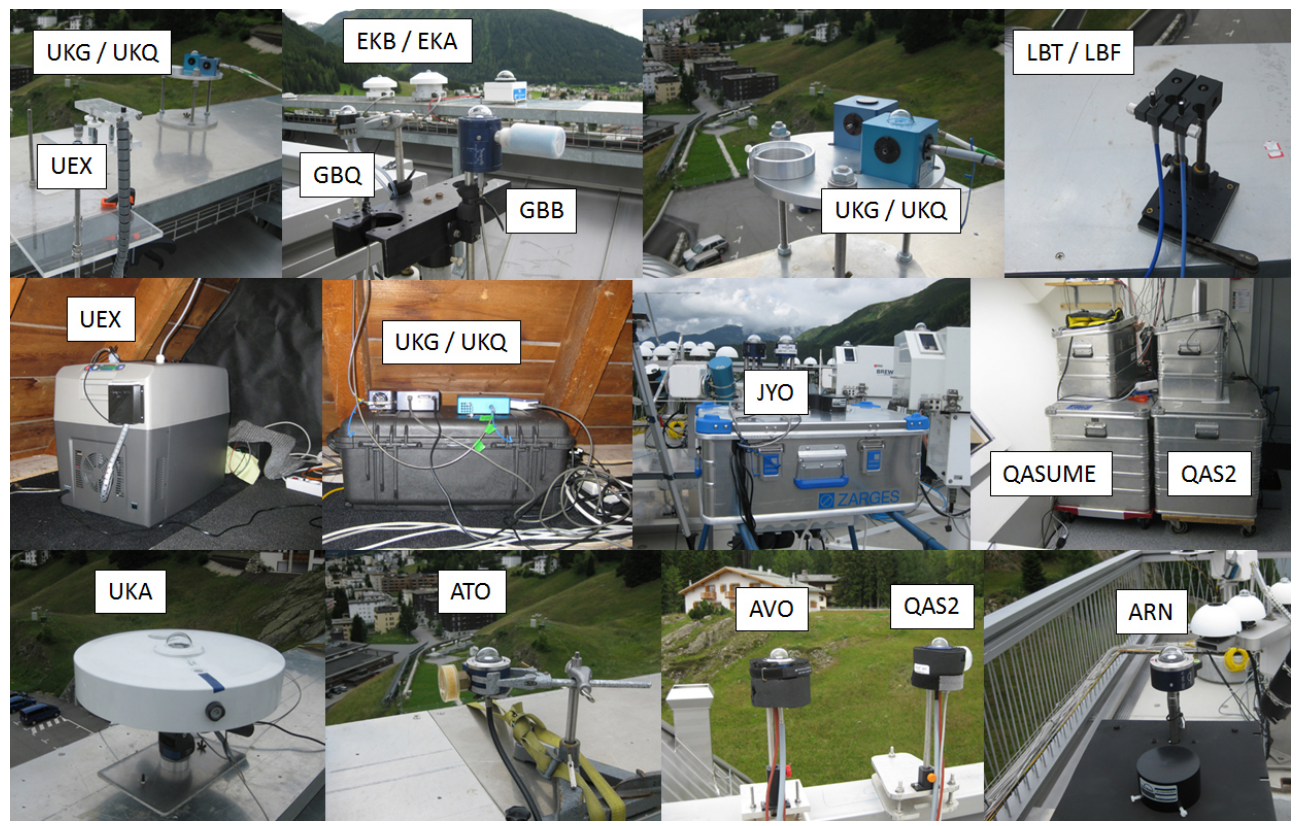

Figure 1. Fourteen array spectroradiometers installed on the measurement platform at PMOD/WRC in Davos, Switzerland. Some of the instruments were in weatherproof boxes on the roof, others were located inside the building under the roof and the entrance optics were connected to the instruments with an optical fibre.

Fourteen ASRMs from the end user community were in operation during the intercomparison; they were set up on the measurement roof at the main building at PMOD/WRC (see Fig. 1). The PMOD/WRC building is located in the valley bottom of Davos surrounded by mountain ridges, which cause a mean shading of diffuse radiation of $5.4 \%$. The setup of the entrance optics on the roof allowed for capturing direct radiation for solar zenith angles (SZAs) at least smaller than $70^{\circ}$. In case of non-weatherproofed instruments, the devices were located under the roof at the PMOD/WRC building in Davos. The ASRMs were operated by different users with different levels of correction methodologies, especially in terms of stray light correction, linearity and temperature stabilization. The different configurations are summarized in Table 1, where the instruments are labelled with their corresponding abbreviations. The scanning spectroradiometers and ASRM instruments are described in the following subsections.

\subsection{Scanning spectroradiometer systems}

\subsubsection{QASUME portable reference spectroradiometer}

In order to compare the results of the end user ASRMs, a reference instrument is required. On behalf of the World Meteorological Organization, the World Calibration Center for UV radiation (WCC-UV) at PMOD/WRC operates the travelling reference spectroradiometer QASUME (Gröbner et al., 2005). This instrument consists of a commercially available Bentham DM-150 double monochromator covering a wave- length range of 290 to $500 \mathrm{~nm}$. The full width at half maximum resolution of the instrument is $0.8 \mathrm{~nm}$. In order to minimize the influence of angular response errors on the global irradiance measurements, specially designed entrance optics from CMS-Schreder (Model UV-J1002) are used (Gröbner et al., 2005), which are connected to the entrance port of the double monochromator through a quartz fibre. Since the instrument is designed for operational outdoor use, the unit is located in a weatherproof and temperature-stabilized box, allowing temperature variations of less than $0.5 \mathrm{~K}$. In order to monitor the stability of the reference spectroradiometer during the operation time, a portable irradiance calibrator is used. The sensitivity of the QASUME spectroradiometer was monitored several times during the intercomparison with three different $250 \mathrm{~W}$ tungsten halogen lamps. The mean responsivity varied by less than $\pm 0.5 \%$, demonstrating that the entire unit was stable during the intercomparison. The temperature of the diffusor head of the entrance optics was kept constant at $28^{\circ} \mathrm{C} \pm 1{ }^{\circ} \mathrm{C}$.

\subsubsection{QASUME II portable reference spectroradiometer}

A second portable reference spectroradiometer, named QASUME II, was developed within the EMRP project "Solar UV", similar to the QASUME spectroradiometer system described above. In analogy to the existing reference, the second portable reference is based on the same double monochromator (Bentham DM-150) in a temperaturestabilized weatherproof housing. In order to improve the sta- 
Table 1. Array spectroradiometer instruments participating at the intercomparison: instruments from the first group are from end users who worked in close collaboration with the EMRP ENV03 project; they are indicated in bold. External instruments from the second group are highlighted in italic. The specific configurations in terms of stray light correction, linearity correction and temperature control are marked.

\begin{tabular}{lllll}
\hline Acronym & Instrument type & $\begin{array}{l}\text { Stray tight } \\
\text { correction }\end{array}$ & $\begin{array}{l}\text { Linearity } \\
\text { correction }\end{array}$ & $\begin{array}{l}\text { Temperature } \\
\text { stabilization }\end{array}$ \\
\hline UKA & APSUS; Ocean Optics QE65000 & & & \\
UKG & B\&Wtek; Glacier X & & \\
UKQ & Ocean Optics; QE65000 & $\sqrt{ }$ & $\sqrt{ }$ \\
GBB & MetCon & $\sqrt{ }$ & $\sqrt{ }$ \\
GBQ & Ocean Optics; USB2000 & $\sqrt{ }$ & \\
ATO & Ocean Optics; USB4000 & $\sqrt{ }$ & $\sqrt{ }$ \\
AVO & Avantes; Ava-Spec ULS2048XL & $\sqrt{ }$ & $\sqrt{ }$ \\
JYO & Jobin Yvon & & & \\
$A R N$ & Avantes; Ava-Spec ULS2048XL & & \\
$U E X$ & Avantes; Ava-Spec ULS2048XL & & \\
$L B F$ & Ocean Optics; USB4000 & & \\
$L B T$ & Ocean Optics; USB2000 & & \\
$E K X$ & EKO Instruments; MS-711 & $\sqrt{ }$ & \\
$E K B$ & EKO Instruments; MS-701 & & \\
\hline
\end{tabular}

bility of the system, a new hybrid detector system based on a silicon photodiode and a photomultiplier H11890 from Hamamatsu was developed (Porrovecchio et al., 2013). The signal of the silicon photodiode is measured with custom made high-sensitive electronics, based on the switched integrator principle while the photomultiplier is operating in counting mode. Both detectors are mounted behind the exit slit of the monochromator to ensure simultaneous measurements by both detectors. Furthermore, QASUME II is equipped with newly developed entrance optics to reduce the uncertainties, due to the deviations of the angular response from the nominal cosine response. The new entrance optics are based on novel quartz-based materials that were studied with model simulations and tested within the EMRP ENV03 project (Pulli et al., 2013).

\subsection{Array spectroradiometers}

\subsubsection{Avantes}

At the intercomparison three ASRMs from the manufacturer Avantes were operated by three different end users. All three instruments were from the type AvaSpec-ULS2048, with a nominal spectral range from 280 to $440 \mathrm{~nm}$ in wavelength. As a detector, a Hamamatsu back-illuminated silicon chargecoupled device (CCD) with 2048 pixels was mounted to the devices. The following instruments participated at the intercomparison:

AVO was additionally equipped with a DUG11X solar blind filter, allowing a transmission of wavelengths between 290 and $380 \mathrm{~nm}$, blocking the parts of the radiation longer than $380 \mathrm{~nm}$ to less than $10^{-5}$. AVO was thoroughly tested and characterized during the EMRP ENV03 project in order to obtain the best possible configuration to measure solar UV radiation. Specifically, the following methods have been applied to this device (see Table 1):

1. Stray light correction algorithm, based on measurements using the pulsed laser for advanced characterizations of spectroradiometers set-up (PLACOS) at the Physikalisch Technische Bundesanstalt (PTB), Braunschweig (Nevas et al., 2014).

2. Linearity correction using the PLACOS set-up (Nevas et al., 2014).

The slit function of AVO varied between $0.53 \mathrm{~nm}$, full width at half maximum at $290 \mathrm{~nm}$ and $0.58 \mathrm{~nm}$, full width at half maximum at $390 \mathrm{~nm}$ wavelength. AVO was connected with a quartz fibre to a Schreder global entrance optic, equipped with the new diffusor material designed during the EMRP ENV03 project, similar to the one used with QASUME II. The instrument was located indoors, without any additional external temperature stabilization.

ARN and UEX were Avantes instruments with the same configuration as AVO but without a DUG11X infrared blocking filter. Therefore, they suffer from out-of-range stray light which cannot be corrected using the in-band stray light correction methods described previously. The instruments covered a wavelength range from 290 to $440 \mathrm{~nm}$ with a spectral bandpass of $0.3 \mathrm{~nm}$ full width at half maximum measured at $325 \mathrm{~nm}$. Each instrument was placed in a temperaturestabilized box connected with a quartz fibre to its entrance optics. ARN used Schreder entrance optics with a shaped diffuser. This diffusor is optimized for cosine error reduction and is from the same type as used for the QASUME reference (Gröbner et al., 2005). On the other hand, UEX was connected to a teflon CC-UV/VIS diffuser provided by Avantes and optimized for cosine error in UV and VIS wave- 
length ranges. The applied methods improving the solar UV measurements were not as thorough as for AVO, i.e. the data of both instruments were corrected neither for stray light nor for linearity (see Table 1).

\subsubsection{Ocean optics}

Six instruments from the manufacturer Ocean Optics were operated by four end users.

UKA and UKQ are QE65000 ASRMs covering the wavelength range between 290 and $400 \mathrm{~nm}$. The QE65000 instrument - labelled here as UKA - is a part of the autonomous portable solar ultraviolet spectroradiometer (APSUS) designed and operated by Public Health England (Hooke et al., 2014). APSUS consists of a weatherproof housing without any additional temperature control. The entrance optics are Ocean Optics $2^{\prime \prime}$ integrating spheres, modified to improve angular response and are protected by a quartz dome. The end user device UKQ is the same QE65000 instrument as UKA, but contrary to UKA, UKQ was designed as a nonweatherproof set-up and without modification of integrating spheres. During operation at the intercomparison, this unit was placed indoors below the measurement platform and connected to an integrating sphere with a quartz fibre. UKA and UKQ had thermoelectrically cooled (TE-cooled) CCD array detectors with a preset cooling temperature of $-10^{\circ} \mathrm{C}$ and were additionally equipped with the solar blind filter U325C. Both instruments were characterized for the slit function at different wavelengths, where the full width at half maximum was between 0.58 at short wavelength and 0.48 at longer wavelength for both UKA and UKQ. Table 1 shows that no stray light or linearity correction was applied to the data of both UKA and UKQ.

The Ocean Optics instrument ATO was from the type USB4000. Similarly to AVO, ATO was thoroughly characterized during the EMRP project, exhibiting the best possible configuration (see Table 1):

1. Stray light correction algorithm based on measurements with a tunable laser facility (PLACOS). The wavelength range covered by ATO is between 200 and $900 \mathrm{~nm}$ covering almost the entire spectrum of the sensitivity of the silicon detector. This implies that mostly an in-range stray light correction was applied (Nevas et al., 2014). However, an out-of-range correction was also applied, based on the method of Kreuter and Blumthaler (2009).

2. Linearity correction based on measurements of the PLACOS set-up.

3. Contrary to AVO, ATO was installed in a temperaturestabilized box ensuring a temperature variation of less than $0.1^{\circ} \mathrm{C}$.

The full width at half maximum of the slit function varied between $1.28 \mathrm{~nm}$ at shorter wavelength and $2.5 \mathrm{~nm}$ at longer wavelength. The entrance optics for ATO were Schreder global optics with a newly developed diffusor based on novel quartz-based materials - as used for QASUME II and AVO - connected to the ATO instrument with a quartz fibre.

An additional Ocean Optics USB4000 ASRM was used for the LBF set-up. LBF was an indoor instrument only, without any temperature stabilization and a non-protected diffusor with an unknown cosine error response. The spectral bandwidth was $2.01 \mathrm{~nm}$, measured at $325 \mathrm{~nm}$. However, the measurement of this slit function was not ideal and resulted in noisy data. The end user operating LBF additionally set up an Ocean Optics USB2000 ASRM (named LBT), again applying the minimum of characterization and calibration methods (Table 1). However, a reliable slit function was obtained, revealing a full width at half maximum of $1.23 \mathrm{~nm}$ at $325 \mathrm{~nm}$. Both instruments covered the wavelength range between 250 and $500 \mathrm{~nm}$.

The last Ocean Optics USB2000 ASRM was GBQ, using a wavelength range between 290 and $850 \mathrm{~nm}$, with a spectral bandpass of $1.1 \mathrm{~nm}$ and a stray light correction (Table 1). Additionally GBQ was temperature-stabilized in a weatherproof box and the entrance optics were an environmentally protected Bentham D7 unit with improved cosine response connected to the instrument with a fibre.

\subsubsection{B\&W Tek}

The instrument UKG from the manufacturer B\&W Tek and the type Glacier $\mathrm{X}$ were operated indoors during the intercomparison. The wavelength covered by the CCD was from 290 to $400 \mathrm{~nm}$. UKG had a TE-cooled CCD array detector with a preset cooling temperature of $14^{\circ} \mathrm{C}$, which is the minimum temperature configurable for this instrument. The device was placed below the measurement platform and connected by a quartz fibre to an Ocean Optics $2^{\prime \prime}$ integrating sphere equipped with a U330C solar blind filter. The full width at half maximum resolution is $0.61 \mathrm{~nm}$ and the wavelength covered by the CCD was from 290 to $400 \mathrm{~nm}$.

\subsubsection{MetCon}

GBB is a CCD ASRM from the manufacturer MetCon. The installed instrument is temperature-stabilized by an air-to-air TEM to $< \pm 0.01{ }^{\circ} \mathrm{C}$ in a weatherproof container. The ASRM consists of a 15-bit diode array instrument with 512 pixels covering a wavelength range between 280 and $700 \mathrm{~nm}$. The spectral bandpass varied between $2.05 \mathrm{~nm}$ at shorter wavelength and $2.16 \mathrm{~nm}$ at longer wavelength. Wavelength calibration was carried out with reference to $\mathrm{Hg}(\mathrm{Ar})$ and $\mathrm{Ne}$ pencil emission lamps. In contrast to the other instruments (except for GBQ) the absolute calibration was carried out with respect to a portable NIST-traceable source, which adds an additional small uncertainty when being compared to the reference instrument, QASUME. The entrance optics are a Schreder cosine optimized diffuser connected to the instrument with a $5 \mathrm{~m}$ fibre. The entire instrument includes a sec- 
ond MetCon diode ASRM, capturing direct solar irradiance, which in turn can be used to retrieve total column ozone. However, this feature was not compared during this intercomparison (but see Smedley et al., 2015 for further instrument details). GBB was also characterized with the PLACOS set-up allowing a stray light correction (Table 1). No linearity correction was applied, although laboratory measurements during the intercomparison gave a non-linearity of $<1.1 \%$ over the full output range.

\subsubsection{Jobin Yvon}

A commercial spectroradiometer from the manufacturer Jobin Yvon VS140 was assembled in the system JYO consisting of a temperature-stabilized box. The nominal wavelength range of the ASRM is between 190 and $625 \mathrm{~nm}$ with a full width at half maximum between 0.64 and $0.76 \mathrm{~nm}$ depending on wavelength. JYO consists of two Schreder entrance optics equipped with two filters allowing transmission of radiation from 280 to $310 \mathrm{~nm}$ for the first entrance optics and from 280 to $400 \mathrm{~nm}$ for the second entrance optics. The spectra were first recorded with one entrance optic, followed by measurements with the second entrance optic. During the post-processing of the data, the spectra from the first measurements were merged to a full spectrum using the wavelength range between 310 and $400 \mathrm{~nm}$ of the second measurements.

\subsubsection{EKO Instruments}

From the manufacturer EKO Instruments, two devices of different types were operated at the intercomparison. The first type was MS-711, labelled EKX. This instrument covered a wavelength range from 300 to $1100 \mathrm{~nm}$, exhibiting a full width at half maximum of $3.35 \mathrm{~nm}$. A stray light correction based on a variable slit function matrix was applied to EKX (see Table 1). The second type of EKO instrument was MS701, labelled as EKB, covering a shorter wavelength range between 300 and $406 \mathrm{~nm}$, with an optical resolution of about $2.84 \mathrm{~nm}$ full width at half maximum bandwidth. The instrument was equipped with its own built-in entrance optics and the housing was temperature-stabilized at $25^{\circ} \pm 5^{\circ}$. No stray light correction was applied to EKB.

\subsection{Data processing}

The quality assessment of the participants' ASRMs was done in the form of a blind intercomparison between QASUME data and data provided by the end users. This means that the QASUME data were not delivered to the ASRM operators until the data analysis for this report was delivered. Furthermore, the post-processing of the data was undertaken by the end users, based on their best knowledge of the sensitivity of their instruments (i.e. counts $\mathrm{s}^{-1}$ per $\mathrm{W} \mathrm{m}^{-2} \mathrm{~nm}^{-1}$ ), wavelength to pixel relationship and in some cases, correction of stray light and non-linearity (see Table 1), without the knowl- edge of the QASUME reference data. The handling of dark count subtraction and the selection of the integration time was also a part of the end users' instrument operation. The measurement interval, including averages of several spectra, could differ for each instrument. The measurement interval and the number of recorded spectra are summarized in Table 2 .

For the following analysis, data from the end users were further processed in order to ensure comparability to the QASUME reference data, obtained wavelength per wavelength with a time step of $1.5 \mathrm{~s}$ between successive wavelengths. First, the solar UV measurements from both QASUME and from ASRMs were analysed with respect to wavelength misalignments relative to a high resolution extraterrestrial solar spectrum developed within the EMRP ENV03 project. The wavelength shift detection algorithm matSHIC is similar to the existing algorithm "ShicRIVM" (Slaper et al., 1995) where the fine structure of the measured solar spectrum is compared with the structure of the high resolution reference spectrum, convolved with the slit function of the ASRM. In particular, the matSHIC algorithm was able to account for a variable slit function over the measured wavelength range and the irregular wavelength grid of ASRM data. The ASRMs which were characterized for the variable slit function by the PLACOS-set-up (Nevas et al., 2014) were UKQ, GBB, GBQ, ATO, AVO and JYO. For the other ASRMs, the wavelength shift detection and the bandwidth homogenization was performed with a constant slit function obtained with a single laser line. The output of the matSHIC algorithm resulted in a solar spectrum with a homogenized wavelength based on the reference spectrum, convolved with a nominal slit function with a full width at half maximum of $1 \mathrm{~nm}$. For the comparison of the data, the same wavelength and grid homogenization were applied to the QASUME reference spectra, resulting in the same wavelength grid and the same nominal slit function as the ASRMs.

Since the ASRM data exhibits a different temporal resolution (i.e. an entire spectrum quasi-simultaneously within seconds) than the data of the scanning spectroradiometer, where $0.25 \mathrm{~nm}$ were sequentially measured every $1.5 \mathrm{~s}$, a time synchronization was applied in the post-processing by the QASUME operators. The observations have been temporarily synchronized by selecting the ASRM spectral irradiance of the wavelength, which is closest in time to the measurement of QASUME. Since the measurement intervals of the ASRMs are longer than $1.5 \mathrm{~s}$ (see Table 2), the corresponding wavelengths are not perfectly matched with the timestamp of the QASUME at a specific wavelength. The effect of nonideal synchronisation may result in some noise of the comparison with the QASUME data at variable atmospheric conditions, such as fast-moving cloud or large solar zenith angles, where the global irradiance may change during a short period of time.

The UV index based on the spectral data is calculated in the post-processing and is defined as the integral of the 
Table 2. Summary of available solar UV data from all ASRMs participating at the intercomparison: ASRMs record the entire spectrum quasiinstantaneously at a specific measurement interval, which is generally shorter than the interval of the scanning spectroradiometer QASUME. The number of spectra which were synchronized to QASUME are indicated. The number of valid days are counted from 1 January 2014.

\begin{tabular}{|c|c|c|c|c|}
\hline Acronym & Number of valid days & Measurement interval & Number of spectra & Synchronized spectra \\
\hline UKA & 8 (days: 191 to 198 ) & $5 \mathrm{~min}$ & 1115 & 140 \\
\hline UKG & 8 (days: 191 to 198 ) & $1 \mathrm{~min}$ & 3602 & 101 \\
\hline UKQ & 8 (days: 191 to 198 ) & $1 \mathrm{~min}$ & 3608 & 90 \\
\hline GBB & 8 (days: 191 to 198 ) & $15 \mathrm{~s}$ & 28814 & 210 \\
\hline GBQ & 8 (days: 191 to 198 ) & $15 \mathrm{~s}$ & 22143 & 175 \\
\hline ATO & 12 (days: 188 to 199 ) & $10 \mathrm{~s}$ & 24361 & 135 \\
\hline AVO & 7 (days: 192 to 198 ) & $30 \mathrm{~s}$ & 10552 & 137 \\
\hline JYO & 4 (days: $194 ; 196$ to 198 ) & $2 \min$ & 192 & 36 \\
\hline$A R N$ & 7 (days: 191 to 197 ) & $30 \mathrm{~s}$ & 8661 & 116 \\
\hline$U E X$ & 7 (days: 191 to 197 ) & $30 \mathrm{~s}$ & 8898 & 105 \\
\hline$L B F$ & 6 (days: 192 to 197 ) & $30 \mathrm{~s}$ & 3884 & 40 \\
\hline$L B T$ & 4 (days: 193,195 to 197 ) & $30 \mathrm{~s}$ & 3392 & 66 \\
\hline$E K X$ & 5 (Days: 196 to 200) & $1 \mathrm{~min}$ & 4805 & 121 \\
\hline$E K B$ & 9 (days: 193 to 201 ) & $1 \mathrm{~min}$ & 8649 & 222 \\
\hline
\end{tabular}

erythema-weighted spectral irradiance. For each day of the intercomparison the UV index is calculated from the data of each individual ASRM. For comparison, all ASRM spectra are synchronized to the QASUME reference as described above. Therefore, one single value of the UV index is available every $30 \mathrm{~min}$. Note that due to the blind intercomparison, for the time synchronization of the spectra and the corresponding calculation of the UV index, the measurements of the ASRMs are processed without any filtering of outliers or other improvement of the data.

\subsection{Measurements and meteorological conditions}

The instruments were partly operated outdoors during 10 July (day of year: 191) to 17 July (day of year 198) 2014. During these days the sky was partly overcast with some clear sky periods resulting in different intensities of global radiation. Figure 2a displays the UV index measured by the QASUME reference during the intercomparison, indicating the variable atmospheric conditions: Between 10 and 15 July the sky was mostly overcast, with some clear sky and interrupted by moving clouds. Between 16 and 17 July the instruments were exposed during mostly cloud-free days. In particular, on 16 July the sky was almost perfectly clear, resulting in a symmetric curve during the day. The UV indices measured by QASUME are also shown to be dependent on the solar zenith angle, indicating the increase of the index at high sun (Fig. 2b). For the analysis of the cosine response of the entrance optics, clear skies from sunrise to sunset for day 197 are needed. The analysis of day 197 regarding cosine response showed that the effect of the cosine response is minor compared to the overall quality of the measurements. Therefore, and also to assess the performance of the instruments, including effects of scattering of clouds and to analyse as much data as possible, overcast days are also included in the study. Furthermore, a comparison between ASRMs and a scanning spectroradiometer under temporarily stable conditions as found during clear skies gives more stable results than under varying cloudy conditions. However, in terms of comparison of weighted quantities such as the UV index, overcast or cloudy skies are less critical than for a spectral analysis.

Some instruments were not continuously in operation during the intercomparison period due to laboratory characterization and mounting and dismounting of non-weatherproof indoor instruments. Therefore, fewer spectra were compared for these instruments. On the other hand, some instruments were operated some days before 10 July and after 17 July. Valid and reliable data from these days were also included in the results of this analysis. The available data sets of all ASRMs are summarized in Table 2.

The reference scanning spectroradiometer QASUME (QAS) was continuously operated during all days of the intercomparison. The measurements started before sunrise (05:00 UTC) and were stopped after sunset (19:00 UTC), covering solar zenith angles between 24 and $85^{\circ}$. The spectra were recorded every $30 \mathrm{~min}$, starting at the full hour. In analogy to QAS, the spectra of the new portable solar UV reference spectroradiometer QASUME II (QAS2) were captured sequentially on a $30 \mathrm{~min}$ interval, synchronized with the QAS schedule by an atomic clock via a network server.

\section{Results}

\subsection{Spectral analysis}

In order to assess the spectral performance of ASRMs, the spectral ratios between the spectra of the specific ASRM and the QASUME reference are calculated for each synchro- 

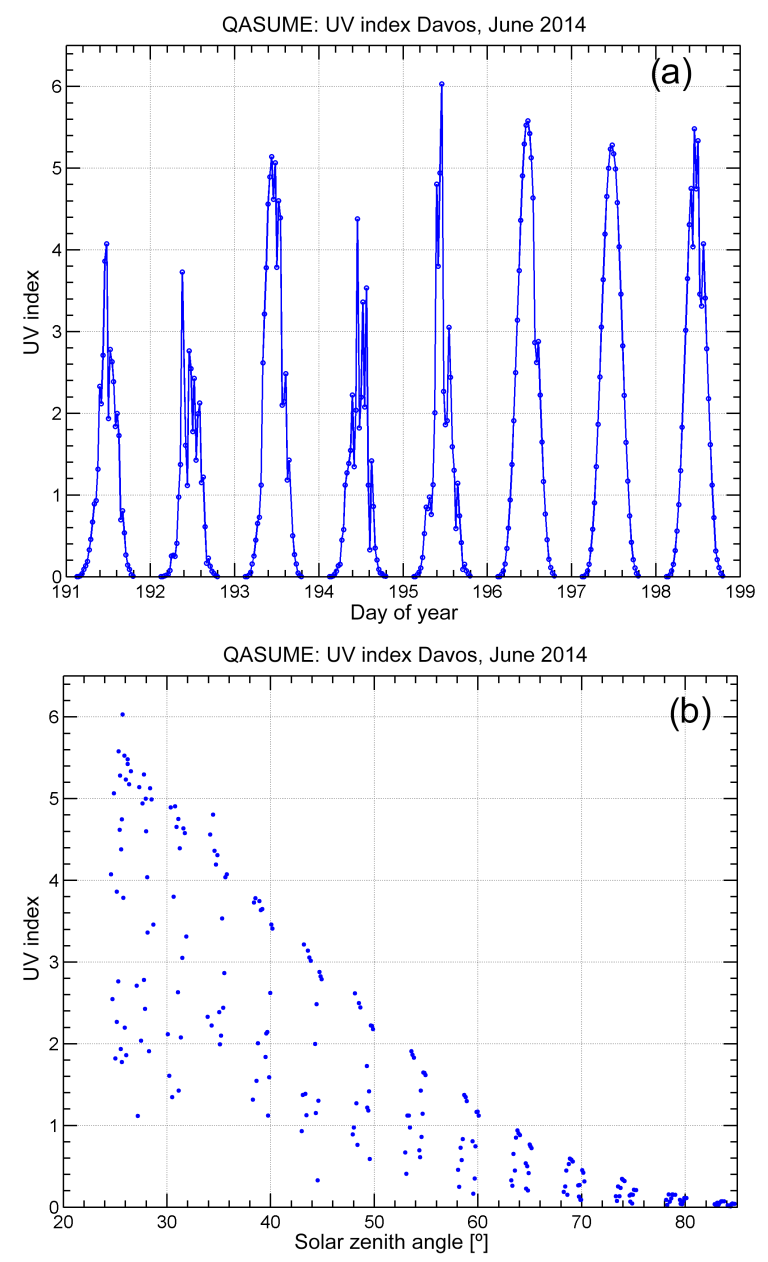

Figure 2. (a) Measurements of the UV index during 10 to 17 July (day of year 191-198) obtained by the reference double monochromator QASUME. (b) The UV index from QASUME as a function of the solar zenith angle.

nized spectrum available from the measurement campaign. The data set covers all solar zenith angles lower than $85^{\circ}$. The number of valid spectra differs for each instrument as summarized in Table 2. Figure 3 shows the average spectral ratios between the ASRMs and QASUME for the wavelength range between 300 and $400 \mathrm{~nm}$ (UV-B and UV-A), even for the ASRMs which measure the spectrum until $500 \mathrm{~nm}$. However, the information on the larger wavelength is shown in Fig. 4 as a dependence on the solar zenith angle. The first eight ASRMs listed in the legend of Fig. 3a (UKA, UKG, UKQ, GBB, GBQ, ATO, AVO and JYO) are instruments that were characterized and calibrated in close collaboration within the EMRP ENV03 project. In the following we will refer to this group of instruments as the "first group". The last six instruments listed in Fig. $3 \mathrm{~b}$ are instruments with less elaborated characterizations prior to the intercomparison and are referred to as the "second group".
As expected, QASUME II (QAS2 - grey line in Fig. 3b) is in very good agreement with the portable reference QASUME, displaying a spectral ratio close to 1 between 300 and $500 \mathrm{~nm}$. The results of QAS2 are typical for wellcharacterized double monochromator instruments, with uncertainties of $5 \%$ or less for wavelengths longer than $300 \mathrm{~nm}$ (Gröbner et al., 2005) and which can be considered as the benchmark for reliable solar UV measurements.

ASRMs: Describing the results of Fig. 3 in more detail, we may highlight that the average spectral ratio of UKA is within this uncertainty band of $0.95-1.05$, when considering the wavelength range between 310 and $400 \mathrm{~nm}$. At shorter wavelengths than $310 \mathrm{~nm}$, however, the ratio deviates from the $5 \%$ benchmark. UKG and UKQ strongly deviate from this result. At $400 \mathrm{~nm}$ the bias is larger than $-15 \%$, rapidly increasing with shorter wavelength. Below $310 \mathrm{~nm}$ the spectral ratio deviates by more than $+10 \%$ due to stray light contribution in the UV-B.

The instrument GBB displays a constant ratio between 320 and $400 \mathrm{~nm}$ of about $-7 \%$. However, even though a stray light correction is applied to the data, the influence of stray light is clearly visible at wavelengths below $310 \mathrm{~nm}$. GBQ shows an almost perfect ratio close to 1 between 340 and $400 \mathrm{~nm}$. The spectral ratio deviates by up to $10 \%$ between 320 and $340 \mathrm{~nm}$ and increases strongly for wavelengths shorter than $320 \mathrm{~nm}$.

The instrument ATO, which is well calibrated and corrected for stray light, still shows an averaged spectral bias of more than $10 \%$ at wavelengths shorter than $310 \mathrm{~nm}$. At wavelengths longer than $310 \mathrm{~nm}$ the difference to QASUME is below $5 \%$ and within the benchmark mentioned above. Similarly to ATO, the instrument AVO was also thoroughly characterized and calibrated. AVO also shows a constant spectral ratio of about $-3 \%$ between 310 and $380 \mathrm{~nm}$. Similarly to ATO, AVO exhibits large biases at wavelength ranges shorter than $310 \mathrm{~nm}$.

The last instrument of the first group is JYO, which is equipped with two entrance optics, separating the UV-B and UV-A part. This instrument shows an average ratio close to 1 . Even though narrow-band filters suppress the stray light contribution at short wavelengths, an impact of stray light can still be observed at wavelengths less than $310 \mathrm{~nm}$.

The instruments of end users, who were not in close partnership with the EMRP ENV03 project (Fig. 3b), deviate significantly more than the instruments mentioned previously. Firstly the ARN and UEX underestimate the solar irradiance at wavelengths between 310 and $400 \mathrm{~nm}$ by 15 to $20 \%$ compared with the QASUME reference instrument. The reason for these large biases is unknown. We may speculate that they are attributed to non-optimized post-processing of the data (e.g. application of the irradiance calibration) by the end users. Below $310 \mathrm{~nm}$ the deviations rapidly increase, exhibiting large differences of more than $20 \%$. A similar underestimation of more than $20 \%$ can be observed for the instruments LBF and LBT. LBF shows large variations in the 

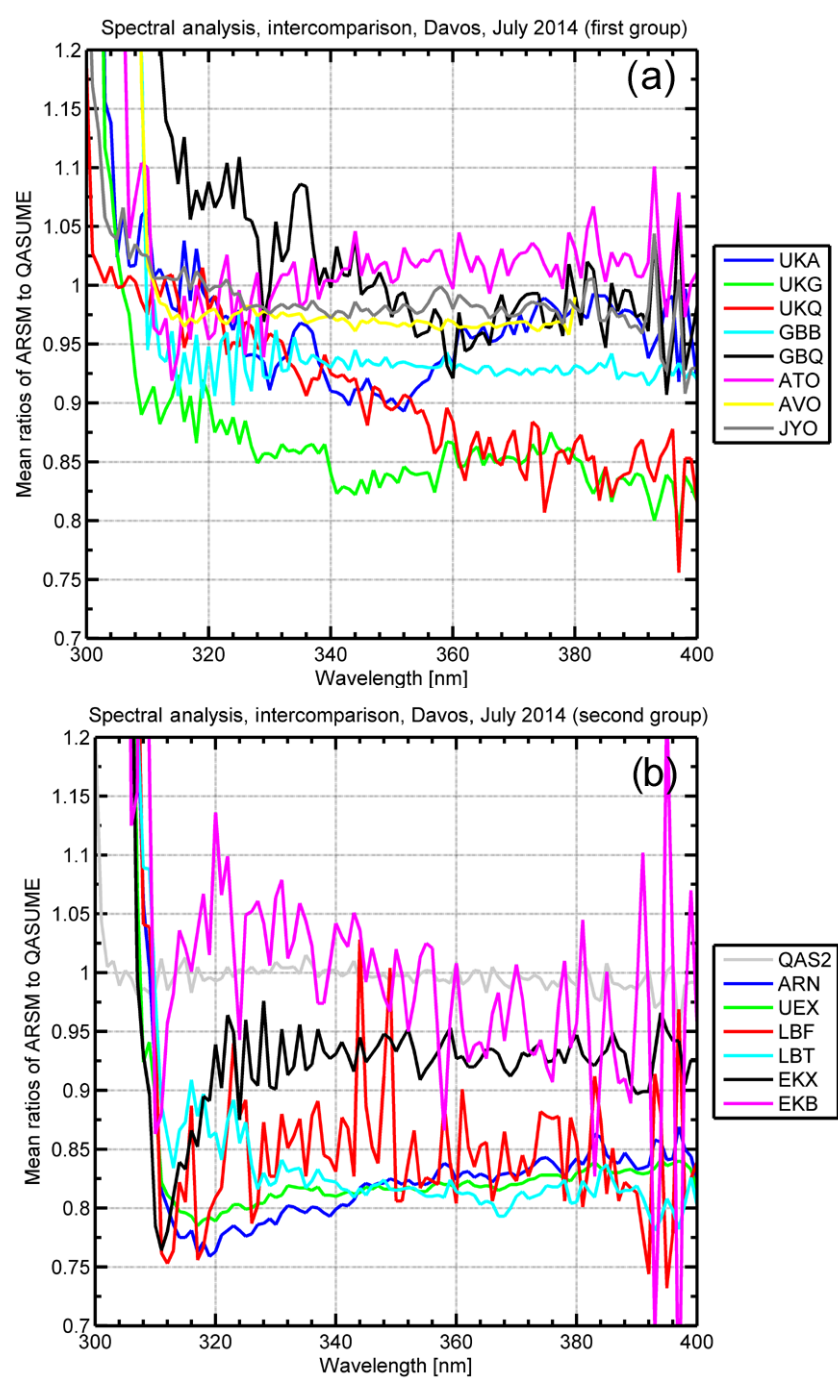

Figure 3. Averaged ratios of the spectral ratio between the QASUME reference and the array spectroradiometers participating at the intercomparison. (a) displays instruments from the first group of devices from end users who were in close collaboration with the EMRP project, while (b) indicates ratios of external end users instruments.

comparison with the QASUME reference. This effect may be due to the error in measured slit function, or the sensitivity of the detector may be too low and therefore, the measurement noise results in scattered data.

The instrument EKX displays a constant underestimation of spectral irradiance of about $-7 \%$ in the wavelength range between 320 and $400 \mathrm{~nm}$. The ratio decreases between 310 and $320 \mathrm{~nm}$ with a rapid increase below $310 \mathrm{~nm}$.

EKB does not show this effect. However, this instrument suffers from large variations in the spectral ratio, with the average lying roughly between -5 and $5 \%$ for wavelengths above $310 \mathrm{~nm}$, with a sharp increase at wavelength ranges lower than $310 \mathrm{~nm}$.
The results in Fig. 3 are averages of spectra including all SZAs. In order to additionally describe the performance of the ASRMs, the ratios between the ASRMs and QASUME are presented in Fig. 4 for different wavelengths with respect to SZA. As indicated in the legend of Fig. 4, the ratios are shown at 305, 310, 320, 350, 400, 450 and $495 \mathrm{~nm}$, averaged over $\pm 2.5 \mathrm{~nm}$. Wavelength ranges below $305 \mathrm{~nm}$ are not shown, since at this wavelength region the ratios to QASUME rapidly increase at large SZAs as well. The figure reveals that the spectral ratios generally also increase at $305 \mathrm{~nm}$ with increasing SZA. Exceptionally, UKQ and JYO show small deviations for SZAs at this wavelength range. At a wavelength of around $305 \mathrm{~nm}$, most of the ASRMs show strong dependence on SZAs, indicating that spectral irradiance in this range is measurable with better agreement to QASUME only at small SZAs. At longer wavelengths, the dependence on SZAs decreases, e.g. for GBB, ARN, UKA, ATO JYO and AVO, where stable ratios can be observed at wavelengths longer than $310 \mathrm{~nm}$. Remarkably, some instruments (e.g. UKQ, UKG) show a decreasing ratio with decreasing SZA at around $60^{\circ}$ and increasing ratios at around $80^{\circ}$. This effect is typical for large cosine errors of the entrance optics. Finally, the instruments EKX and EKB underestimate the global irradiance at wavelengths between 305 and $310 \mathrm{~nm}$, while at longer wavelengths the ratios are within $10 \%$ for all SZAs.

\subsection{UV index}

The standard parameter for disseminating UV exposure on human health is the UV index (WMO, 1998). Therefore, and as a product of the spectral measurements, the UV index was calculated for each ASRM as well as for the QASUME reference (Fig. 2). The temporal changes of the UV index over the entire intercomparison period reveal large variations of the UV index obtained by ASRMs. Some instruments overestimate the index by about 1 unit, while others detect values which clearly do not correspond to realistic values observed during these days. In particular, instruments in the second group show many outliers, with UV indices of larger than 11 (specific graph not shown).

Analysing the daily course of the UV index in more detail, the ratios of UV indices over the QASUME reference, as a function of the solar zenith angle, are presented in Fig. 5. The statistics of the performance of the ASRMs for different ranges of SZAs are listed in Table 3 and described in the following.

The best averaged performance from the first group of instruments was obtained by the instrument UKA, showing a deviation to QASUME of less than $1 \%$ mean value (ratio of 0.993 , Table 3) for all solar zenith angles lower than $70^{\circ}$. However, the single measurements deviate by $\pm 5 \%$ $( \pm 0.048$, Table 3$)$ from the mean value. The instrument UKG, operated by the same end user as UKA, showed an underestimation by a factor of $0.94 \pm 0.03$ for small SZAs 


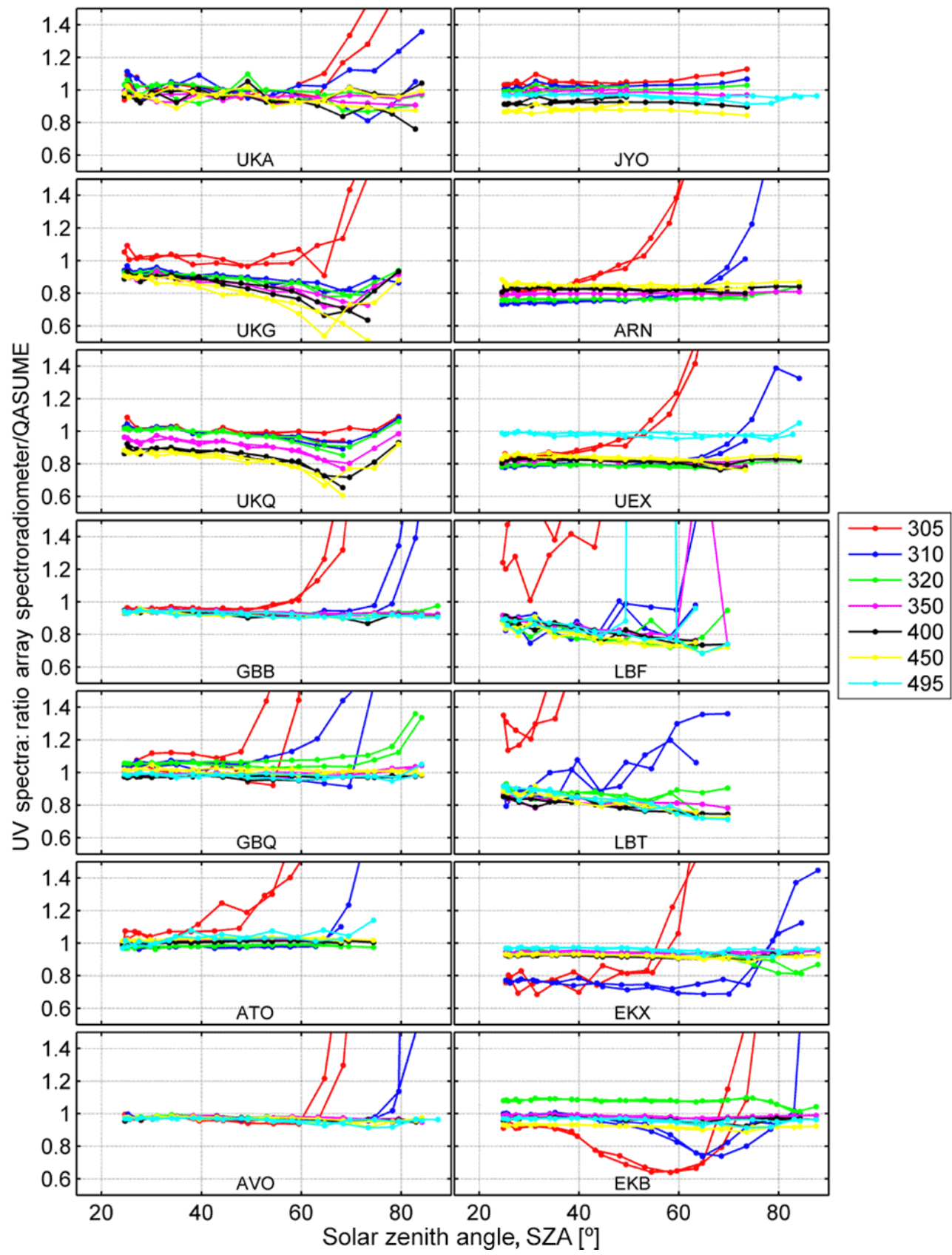

Figure 4. The ratios of spectral global UV irradiance derived from all array spectroradiometers to the QASUME reference spectroradiometer. The ratios are calculated from all available data of the campaign and are shown as a function of solar zenith angle and for selected wavelength between 305 and $495 \mathrm{~nm}$.

and $0.923 \pm 0.03$ for the range of SZAs less than $70^{\circ}$. UKQ performed similarly to UKA, showing an averaged bias of less than $1 \%$ for SZAs less than $30^{\circ}$ (Table 3). At larger SZAs the performance of UKQ ratios decreased to about $0.979 \pm 0.023$.

Analysing the results of GBB for SZAs smaller than $70^{\circ}$ an averaged bias of 1.05 is shown, while for SZAs smaller than $50^{\circ}$ the agreement with QASUME is within about 0.98 .
Remarkably, the deviation from this mean value is in the order of \pm 0.08 . Much larger overestimations and deviations between single measurements are shown for GBQ, where the averaged ratios are around $1.5 \pm 0.27$ even for SZAs smaller than $50^{\circ}$.

The thoroughly characterized ASRM ATO shows a very large overestimation of the UV index by a factor of 


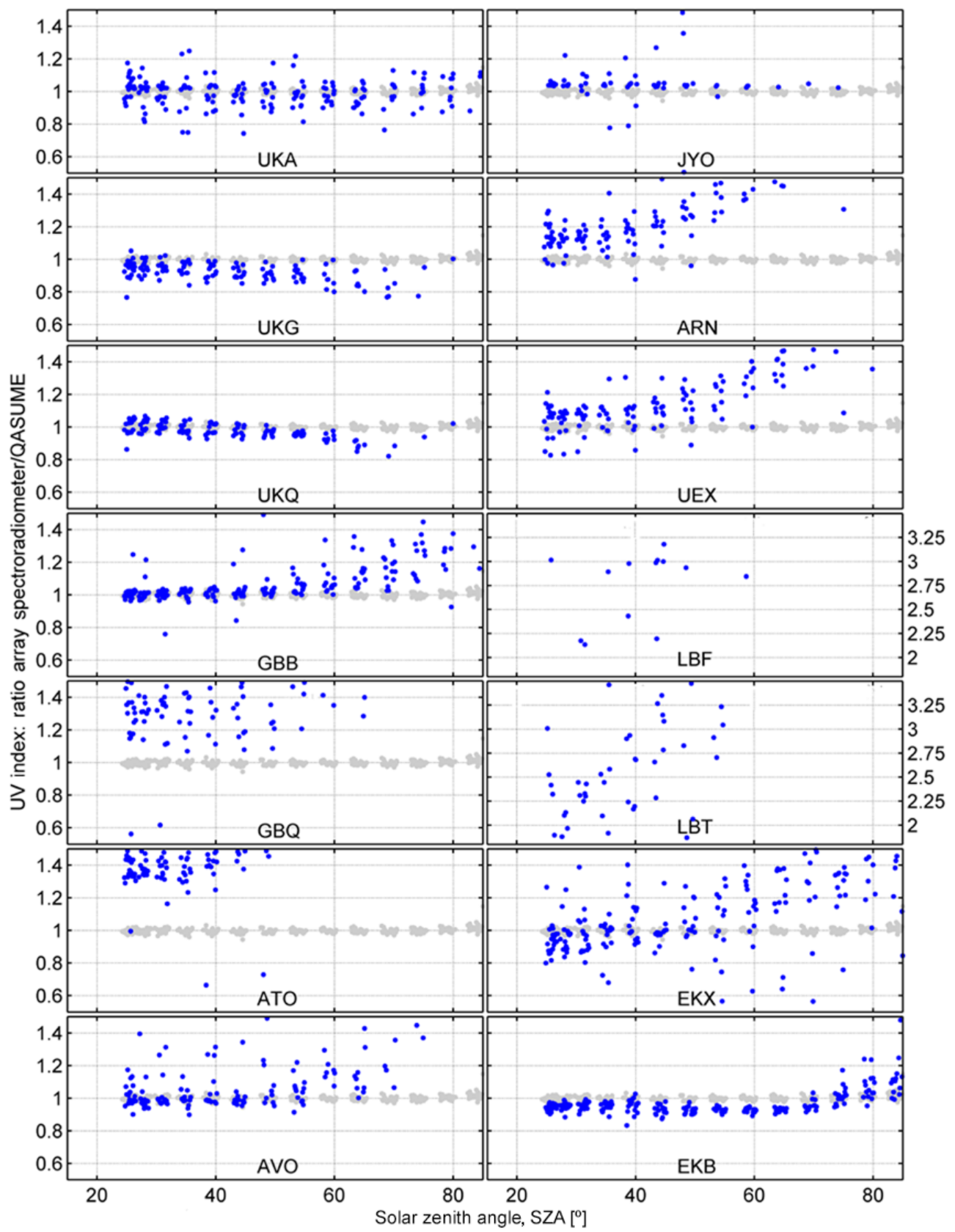

Figure 5. The ratios of UV indices derived from all array spectroradiometers (blue points) to the QASUME reference spectroradiometer, as a function of solar zenith angle. The grey points indicate the ratio between QASUME and QASUME II in order to display the determination of the UV index with double monochromator instruments. The $y$ axis of LBF and LBT has been adjusted to display the results.

$1.489 \pm 0.145$ for $\mathrm{SZA}<50^{\circ}$ and an even larger factor of $1.8 \pm 0.49$ for SZAs between 24 and $70^{\circ}$.

Similarly to ATO, the instrument AVO was thoroughly characterized and evaluated during the EMRP ENV03 project. The UV indices, however, are comparable to the reference when considering measurements recorded at SZAs smaller than $50^{\circ}$. In this case, the UV index is on average within $4 \%$, while most of the single values deviate about $6 \%$ from the average, with some significant outliers.
The last instrument from the first group, JYO, is equipped with two entrance optics and different filters to reduce stray light. This instrument shows an average ratio of about 1.03 for all SZAs smaller than $70^{\circ}$ during the three days of operation. Most of the observations deviate less than 2 to $3 \%$ from the mean value and are close to the ratios between QASUME II and QASUME. However, several outliers in the data are unexplained. 
Table 3. Statistics of ratios between QASUME and all array spectroradiometers regarding the UV index: the numbers indicate the averaged ratios within the selected range of solar zenith angles (SZAs), with their corresponding deviation from the mean.

\begin{tabular}{lccc}
\hline Acronym & $\mathrm{SZA}<30^{\circ}$ & $\mathrm{SZA}<50^{\circ}$ & $\mathrm{SZA}<70^{\circ}$ \\
\hline UKA & $1.02 \pm 0.04$ & $1.00 \pm 0.51$ & $0.99 \pm 0.48$ \\
UKG & $0.95 \pm 0.03$ & $0.94 \pm 0.23$ & $0.92 \pm 0.03$ \\
UKQ & $1.01 \pm 0.02$ & $0.99 \pm 0.02$ & $0.98 \pm 0.02$ \\
GBB & $0.98 \pm 0.09$ & $0.99 \pm 0.08$ & $1.05 \pm 0.19$ \\
GBQ & $1.38 \pm 0.15$ & $1.51 \pm 0.27$ & $2.05 \pm 0.69$ \\
ATO & $1.49 \pm 0.18$ & $1.49 \pm 0.15$ & $1.88 \pm 0.50$ \\
AVO & $1.02 \pm 0.04$ & $1.04 \pm 0.07$ & $1.29 \pm 0.58$ \\
JYO & $1.05 \pm 0.02$ & $1.03 \pm 0.01$ & $1.03 \pm 0.10$ \\
$A R N$ & $1.12 \pm 0.04$ & $1.17 \pm 0.08$ & $1.28 \pm 0.16$ \\
$U E X$ & $1.04 \pm 0.05$ & $1.07 \pm 0.05$ & $1.16 \pm 0.09$ \\
$L B F$ & $1.96 \pm 0.74$ & $3.86 \pm 1.04$ & $4.76 \pm 1.31$ \\
$L B T$ & $2.13 \pm 0.26$ & $2.61 \pm 0.39$ & $3.01 \pm 0.61$ \\
$E K X$ & $0.93 \pm 0.12$ & $0.97 \pm 0.14$ & $1.11 \pm 0.37$ \\
$E K B$ & $0.95 \pm 0.01$ & $0.94 \pm 0.01$ & $0.94 \pm 0.04$ \\
\hline
\end{tabular}

Describing the ASRMs from the second group which were not characterized during the framework of the EMRP ENV03 project significant outliers are visible. The instrument ARN overestimates the UV index by more than $1.3 \pm 0.16$ for all SZA smaller than $70^{\circ}$. Even for small SZAs, the UV index is overestimated by more than $12 \% \pm 4 \%$. The instrument from the same manufacturer, UEX, displays mean ratios over all SZAs to $7 \% \pm 5 \%$ averaged for SZAs smaller than $50^{\circ}$, similarly to ARN.

For the instruments LBT and LBF, the range of the $y$ axis had to be adjusted to display the ratios between QASUME and these instruments (Fig. 5). LBT shows an overestimation by a factor of $2.12 \pm 0.25$, even for small SZAs, and the overestimation of LBF is in a similar range.

The instruments from the manufacturer EKO (EKB and EKX) exhibit the best average performance of the second group. In particular, EKB shows a stable average performance of about $0.94 \pm 0.03$ for SZAs smaller than $70^{\circ}$. The averaged ratios between EKX compared to QASUME are $0.977 \pm 0.014$ averaged for all SZAs $<50^{\circ}$, exhibiting an increasing trend with increasing SZA.

In summary, the results reveal that estimates of the UV indices by ASRMs strongly depend on the solar zenith angle and most of the single spectra deviate more than $5 \%$ from the mean value at large SZA (Fig. 5), where stray light contribution plays a major role in the wavelength region below $310 \mathrm{~nm}$. Figure $2 \mathrm{~b}$ shows, however, that with large SZAs, the absolute value of the UV index is low and the impact of large over- or underestimations therefore result in a low absolute deviation of the index.

For comparison of the ASRM performance, the grey points of Fig. 5 display the performance of the QASUME II scanning double monochromator. The results reveal that the agreement of QASUME II is close to 1 for all measured spectra from sunrise to sunset, exhibiting a deviation from this average of less than $2 \%$, even for large SZAs.

\section{Discussion}

The results described above will be discussed in more detail in order to address some specific issues regarding the performance of the ASRMs. It can clearly be seen that during a whole day of measurements, from sunrise to sunset, all ASRMs are unable to detect spectral global solar UV radiation in the full wavelength range between 300 and $400 \mathrm{~nm}$ (or $500 \mathrm{~nm}$ respectively) with the same quality as QASUME (Figs. 3-5).

The UV index can be detected by some instruments with average ratios compared to the reference of about $5 \%$ for SZAs smaller than $50^{\circ}$ (e.g. UKA, UKG, UKQ, GBB, ATO, AVO, EKX - Table 3). However, all ASRMs overestimate the global irradiance below $310 \mathrm{~nm}$ (Figs. 3 and 4) for all SZAs. This wavelength range contributes significantly to the UV index and one may assume that due to this fact, all UV indices may also be overestimated. This interesting finding can be exemplified by instruments UKG and UKA. Remarkably, the erythema-weighted spectra in terms of the UV index of these instruments is close to $1 \pm 0.05$ for all SZAs, (Fig. 5, Table 3). The spectral ratios of both instruments, however, show a large underestimation, in particular, in the UV-A part of more than $15 \%$ and an overestimation in the UV-B part of more than $20 \%$. This indicates that the overestimation in the UV-B part compensates the underestimation of the UV-A part, resulting in a reasonable agreement for UV index. However, a large deviation of the single UV index estimates from the mean value (Fig. 5) is found for this instrument. This may indicate that the compensation effect may not account for the different atmospheric conditions.

The second instrument determining the UV index within less than 5\% $\pm 2 \%$ compared to QASUME is JYO (Fig. 5). This instrument also shows an accurate spectral ratio close to 1 at wavelength around $305 \mathrm{~nm}$ with decreasing ratio down to 0.85 at longer wavelengths (Fig. 4). The stray light contribution in the UV-B part (around $305 \mathrm{~nm}$ ) is smaller than for all other ASRMs and seems to compensate the underestimation at longer wavelengths resulting in a correct UV index with small deviations at all SZAs (Fig. 5). Remarkably, stray light correction was not applied to the data of this instrument and the influence of the stray light does not affect the UV index substantially. Therefore, we may conclude that stray light reduction using filters improves the measurements of ASRMs as well as stray light correction, at least for the determination of the UV index. This finding again highlights the importance of stray light rejection as stated in earlier publications (e.g. Edwards and Monks, 2003).

Instruments which are well characterized during the EMRP ENV03 project and which show good spectral agree- 
ment to the QASUME reference, in particular in the UV-A part, such as GBB, ATO and AVO, can still show large overestimations of the UV index. Regarding the spectral analysis (Figs. 3 and 4), all of these instruments show large overestimations in the UV-B part, resulting in a higher value of the weighted integral of the UV index. All of these instruments (except JYO discussed above) applied stray light correction based on line spread functions of tuneable laser facilities (Nevas et al., 2014); however, the stray light reduction is obviously not sufficient to achieve a reliable UV index. For example, the instrument ATO, covering a wavelength range between 290 and $900 \mathrm{~nm}$, applied mostly in-range stray light correction (Nevas et al., 2014), which did not improve the determination of the UV index sufficiently, even for small SZAs.

The reason is that after stray light correction, the remaining spectral noise for a particular instrument may be greater than any detectable signal, particularly at the shortest wavelengths in the UV-B range, and if for the calculation of the UV index, the integration carried out includes these wavelengths (from $290 \mathrm{~nm}$ ), then a significant overestimation of the UV index will result, as seen in Fig. 5 for ATO. Therefore it may be necessary to add another layer of post-processing where the cut-off wavelength in the UV-B range is calculated for each spectrum and the irradiance below this wavelength is set to zero. The determination of the cut-off wavelength is based on a linear fit to a logarithmic plot of the irradiance in the shortest UV-B wavelength range relative to the longer wavelengths. This additional post-processing was originally not applied to the ATO data and therefore, the UV index shown in Fig. 5 is significantly overestimated. When it is carried out, the UV index becomes significantly smaller and more accurate (Fig. 6). In general, whether such a step is necessary depends on the instrument and its specific noise equivalent irradiance. Due to the selected measurement interval (see Table 2) and the corresponding small noise equivalent irradiance, it is assumed that the measurement procedure of the other instruments may be improved to reduce the noise of the measurements. It should be highlighted that sophisticated post-processing methods, e.g. including radiative transfer models simulating the noisy spectrum at lower wavelength, may significantly improve products derived from the spectrum, such as the UV index. However, the aim of the study is to investigate the quality of the raw spectral measurements rather than to improve applications or products derived from the measurements.

Contrary to ATO, AVO employed a solar blind filter to reduce the impact of the longer wavelength radiation and additionally applied in-range stray light correction. This instrument was able to detect UV indices for solar zenith angles $<50^{\circ}$ within $5 \%$ uncertainty, indicating that optical filters may improve the stray light reduction better than a stray light correction method in the post-processing of the data.

The other instruments, mainly those end user instruments which were not embedded in the characterization and cali-

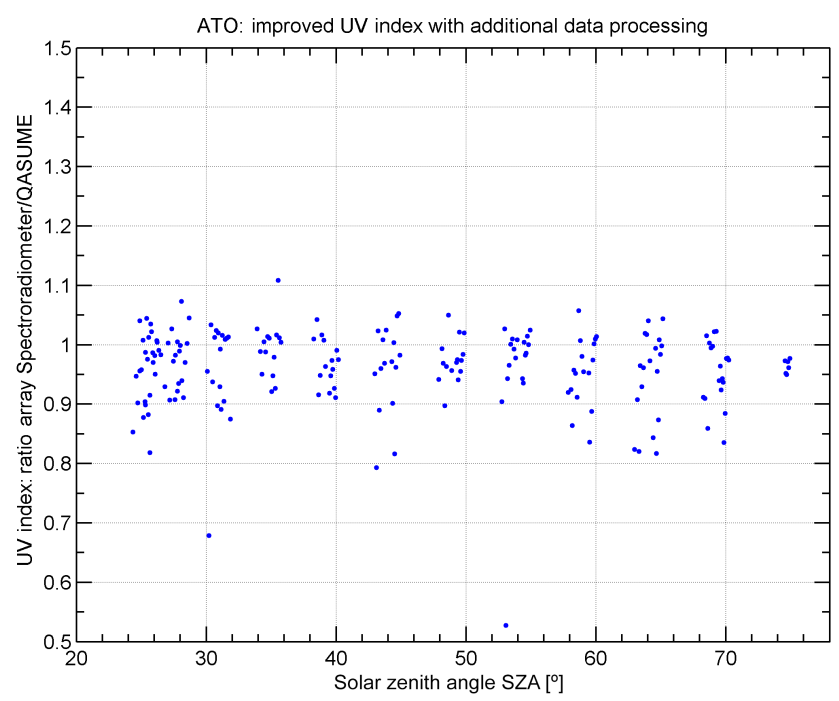

Figure 6. The ratio of UV indices derived from the ATO array spectroradiometer to the QASUME reference spectroradiometer, as a function of solar zenith angle. The UV indices were recalculated after additional post-processing of the original data, to correct for unrealistic values at short wavelength in the UV-B part of the spectrum.

bration procedure since the beginning of the EMRP ENV03 project, showed a general performance, which is insufficient to determine either the UV index or the spectral measurement of global irradiance or both. However, additional data postprocessing shown as exemplary for ATO, but customized for the other instruments may also improve estimations of the UV index for these instruments.

Overall, it is important to keep in mind that the main advantage of spectral instruments is the ability to afterwards apply any desired weighting function to calculate weighted irradiances, where the UV index is one example of a weighting function. Therefore, the main quality criterion for ASRMs is the good agreement of spectral irradiance with the reference. Then any weighted quantity will be in good agreement too, even with different weighting functions such as erythema or vitamin $\mathrm{D}$ formation.

\section{Conclusion}

The intercomparison of 14 ASRMs in July 2014 at the measurement platform at the PMOD/WRC building in Davos produced the following results:

- The tested commercially available ASRMs need thorough calibrations and characterizations for solar UV measurements in order to reliably measure solar UV radiation. Instruments which were in close collaboration with the EMRP ENV03 generally performed better than external end user devices with less detailed characterisations. 
- Some well-characterized instruments are able to determine the UV index within $5 \%$, compared to the QASUME reference for SZAs less than $50^{\circ}$.

- Almost all tested instruments suffer from stray light contribution at wavelengths shorter than $310 \mathrm{~nm}$ for all sampled atmospheric conditions. At smaller SZAs the ratios between QASUME and a few instruments were between 0.95 and 1.05 if a stray light reduction is applied, either with stray light correction, according to Nevas et al. (2014), or with optical filters.

- None of the participating ASRMs are able to detect solar UV radiation at wavelengths shorter than $310 \mathrm{~nm}$ with the same quality as double monochromator instruments.

- The assessment based on this study does not recommend a specific instrument or manufacturer which exhibits a superior quality of solar UV measurement.

In summary, commercially available ASRMs are limited in measuring global solar UV radiation. To further improve the quality of spectral solar UV measurements by ASRMs, additional efforts are needed. Based on the results of the EMRP project ENV03, the World Calibration Center for UV radiation at PMOD/WRC is further developing ASRM characterization techniques in order to improve the quality of solar UV radiation measurements worldwide.

Acknowledgements. The research work leading to this article was carried out within the EMRP ENV03 project, "Traceability for surface spectral solar ultraviolet radiation.” The EMRP is jointly funded by the EMRP participating countries within EURAMET and the European Union.

The authors further thank the project partners of the EMRP ENV03 project "Solar UV", Steven van den Berg, Peter Blattner, Omar El Gawhary, Petri Kärhä and Saulius Nevas, for the characterization and calibration services during the project. Many thanks are for María Luisa Cancillo (University of Extremadura), Jose Antonio Bogeat (INTA) and Alexander Los (EKO instruments) for additional operation of instruments during the intercomparison. Furthermore, all colleagues of PMOD/WRC are acknowledged for technical and administrative assistance during the intercomparison in Davos.

A. Serrano and J. M. Vilaplana are thankful for the research projects CGL2011-29921-C02 and CGL2014-56255-C2, granted by the Ministerio de Economía y Competitividad from Spain.

Edited by: G. Phillips

\section{References}

Anders, A., Altheide, H. J., Knälmann M., and Tronnier, H.: Action spectrum for erythema in humans investigated with dye lasers, Photochem. Photobiol., 61, 200-205, 1995.

Bais, A. F., Gardiner, B. G., Slaper, H., Blumthaler, M., Bernhard, G., McKenzie, R., Webb, A. R., Seckmeyer, G., Kjeldstad, B., Koskela, T., Kirsch, P., Gröbner, J., Kerr, J. B., Kazadzis, S., Leszczynski, K., Wardle, D., Brogniez, C., Josefsson, W., Gillotay, D., Reinen, H., Weihs, P., Svenoe, T., Eriksen, P., Kuik, F., and Redondas, A.: SUSPEN intercomparison of ultraviolet spectroradiometers, J. Geophys. Res., 106, 12509-12526, 2001.

Bernhard, G., Mayer, B., Moise, A., and Seckmeyer, G.: Measurements of spectral solar UV irradiance in tropical Australia, J. Geophys. Res., 102, 8719-8730, 1997.

Bittar, A. and McKenzie, R. L.: Spectral ultraviolet intensity measurements at 458S: 1980 and 1988, J. Geophys. Res., 95, 55975603, 1990.

Blumthaler, M., Gröbner, J., Egli, L., and Nevas, S.: A guide to measuring solar UV spectra using array spectroradiometers, Proc. AIP Conference, 6-10 August 2012, Dahlem Cube, Free University, Berlin, 1531, 805, doi:10.1063/1.4804892, 2013.

Coleman, A., Sarkany, R., and Walker, S.: Clinical ultraviolet dosimetry with a CCD monochromator array spectroradiometer, Phys. Med. Biol., 53, 5239-5255, 2008.

Edwards, G. D. and Monks, P. S.: Performance of a singlemonochromator diode array spectroradiometer for the determination of actinic flux and atmospheric photolysis frequencies, J. Geophys. Res.-Atmos., 108, 8546, doi:10.1029/2002JD002844, 2003.

Gröbner, J., Schreder, J., Kazadzis, S., Bais, A. F., Blumthaler, M., Görts, P., Tax, R., Koskela, T., Seckmeyer, G., Webb, A. R., and Rembges, D.: Traveling reference spectroradiometer for routine quality assurance of spectral solar ultraviolet irradiance measurements, Appl. Optics, 44, 5321-5331, 2005.

Gröbner, M., Gröbner, J., and Hülsen, G.: Quantifying UV exposure, vitamin D status and their relationship in a group of high school students in an alpine environment, Photochem. Photobiol. Sci., 14, 352, doi:10.1039/c4pp00324a, 2015.

Hofzumahaus, A., Kraus, A., and Müller, M.: Solar actinic flux spectroradiometry: a technique for measuring photolysis frequencies in the atmosphere, Appl. Optics, 38, 4443-4460, doi:10.1364/AO.38.004443, 1999.

Holick, M., Bouillon, R., Eisman, J., Garabedian, M., Kleinschmidt, J., Suda, T., Terenetskaya, I., and Webb, A.: Action Spectrum for the production of previtamin D3 in human skin, Commission Internationale de l'Éclairage, Technical Rep., ISBN: 3901906 50 9, 2006.

Hooke, R., Pearson, A., and O'Hagan, J.: Autonomous Portable Solar Ultraviolet Spectroradiometer (APSUS) - a new CCD spectrometer system for localised, real-time solar ultraviolet (280-400 nm) radiation measurement, Photochem. Photobiol., 90, 903-910, doi:10.1111/php.12251, 2014.

Kazadzis, S., Bais, A. F., Balis, D., Zerefos, C. S., and Blumthaler, M.: Retrieval of downwelling UV actinic flux density spectra from spectral measurements of global and direct solar UV irradiance, J. Geophys. Res.-Atmos., 105, 4857-4864, doi:10.1029/1999JD900400, 2000. 
Kreuter, A. and Blumthaler, M.: Stray light correction for solar measurements using array spectrometers, Rev. Sci. Instrum., 80, 096108, doi:10.1063/1.3233897, 2009.

Mayer, B. and Kylling, A.: Technical note: The libRadtran software package for radiative transfer calculations - description and examples of use, Atmos. Chem. Phys., 5, 1855-1877, doi:10.5194/acp-5-1855-2005, 2005.

McKinlay, A. F. and Diffey, B. L.: A reference action spectrum for ultraviolet induced erythema in human skin, Commission Internationale de l'Eclairage, 6, 17-22, 1987.

Nevas, S., Gröbner, J., Egli, L., and Blumthaler, M.: Stray light correction of array spectroradiometers for solar UV measurements, Appl. Optics, 53, 4313-4319, doi:10.1364/AO.53.004313, 2014.

Porrovecchio, G., Smid, M., Gröbner, J., Rajteri, M., Portesi, C., Nield, K., and Egli, L.: New Detection Systems for UV Solar Reference Scanning Spectroradiometers, AIP Conference Proceedings, 1531, 837, doi:10.1063/1.4804900, 2013.

Pulli, T., Kärhä, P., and Ikonen, E.: A method for optimizing the cosine response of solar UV diffusers, J. Geophys. Res.-Atmos., 118, 7897-7904, 2013.
Seckmeyer, G., Bais, A., Blumthaler, M., Druke, S., Kiedron, P., Lantz, K., McKenzie, R., and Riechelmann, S.: Instruments to measure solar ultraviolet radiation Part 4: Array Spectroradiometers, WMO/GAW No.191, World Meteorological Organization, Geneva, 2010.

Slaper, H., Reinen, H. A. J. M., Blumthaler, M., Huber, M., and Kuik, F.: Comparing ground level spectrally resolved UV measurements from various instruments: A technique resolving effects of wavelength shifts and slit widths, Geophys. Res. Lett., 22, 2721-2724, 1995.

Smedley, A. R. D., Kift, R. C., and Webb, A. R.: Assessment of a dual-channel array spectrometer for ground-based ozone retrievals, J. Atmos. Ocean. Technol., 32, 1464-1477, 2015.

WMO: Report of the WMO-WHO Meeting of Experts on Standardization of UV Indices and their Dissemination to the Public, Les Diablerets, Switzerland, 21-24 July 1997, World Meteorological Organization Golbal Atmospheric Watch No. 127, WMO/TDNo. 921, 1998. 